\title{
Effect of Angle of Rotation for Fiber Aligned in a Composite Material Wall of Inclined Enclosure on Heat Transfer
}

\author{
Manal AL-Hafidh, Ph.D \\ Department of Mechanical \\ Engineering \\ University of Baghdad
}

\author{
Haithem H. Muhammad \\ Department of Mechanical \\ Engineering \\ University of Baghdad
}

\author{
Ban B. Jawad \\ Department of Mechanical \\ Engineering \\ University of Baghdad
}

\begin{abstract}
A numerical study has been conducted for natural convection of air in a three dimensional inclined annulus enclosure. This study wills exam the effect of fibres filler in composite material of inclined enclosure on heat transfer. Two types of optimization will be performed in terms of effective thermal conductivity: minimization and maximization of thermal conductivity. The annulus material is made of Graphite/epoxy laminated composite materials. The annulus enclosure is filled with porous media between two inclined concentric cylinders with 12 fins attached to the inner cylinder. The system is under steady state condition and constant walls temperature boundary condition. The parameters affected on the system are modified Rayleigh number $\left(10 \leq \mathrm{Ra}^{*} \leq 500\right)$ and the annulus inclination angle $\delta\left(0^{\circ}, 30^{\circ}, 45^{\circ}, 60^{\circ}\right.$ and $\left.90^{\circ}\right)$. For all parameters, results showed that the average $\mathrm{Nu}$ number decrease with the increase of $\delta$ for high values of $\mathrm{Ra}^{*}$ and increases with an increase in modified Rayleigh number but for low values of $\mathrm{Ra}^{*}$, the effect of $\delta$ on the average $\mathrm{Nu}$ will be low. The deviation between the average $\mathrm{Nu}$ for the maximization and minimization of the thermal conductivity is equal to $5.1 \%$ for horizontal annulus $\delta=0^{\circ}$ and $10 \%$ for vertical annulus $\delta=90^{\circ}$. A correlation for the average Nusselt number in terms of $\mathrm{Ra}^{*}$ and $\delta$, has been developed for the outer cold cylinder.
\end{abstract}

\section{Keywords}

Natural convection, inclined annulus, laminar flow, porous media, graphite/epoxy, composite material.

\section{INTRODUCTION}

Composite materials are used in various designs to improve the characteristic of various constructions and reduce their weight. Researchers and engineers all over the world studied the properties of these materials and the problems of obtaining structural elements based upon them. Composite materials have been developed for a wide range of industrial applications, including piping, pressure vessels, fluid reservoirs, aerospace components, and naval structures. Composite materials have many favorable properties, including high strength to- weight ratios and high corrosion resistances. Many studies have investigated the mechanical and thermo mechanical properties of composite laminate. These materials are used in various designs to improve the characteristic of various constructions and reduce their weight. The properties of these materials and the problems of obtaining structural elements based upon them have been studied by researchers and engineers all over the world [1]. Any property of a composite which is made of two (or more) materials has the value which is the resultant of a few factors.
Obviously, the most important are the values of a certain property of each constituent material. However, one of the factors that also influence the resultant value of a property of a composite as a whole is its geometrical structure. Such resultant properties are commonly called effective properties of a composite. Temperature is the most important of all environmental factors affecting the behavior of composite materials, mainly because composites are rather sensitive to temperature and have relatively low effective thermal conductivity. For instance, advanced composites for engineering applications are characterized with low density providing high specific strength and stiffness, low thermal conductivity resulting in high heat insulation, and negative thermal expansion coefficient allowing us to construct hybrid composite elements that do not change their dimensions under heating. The fields of composite applications are diversified [2].

[3] Prepared nanocomposites by dispersing three different grades of graphite particles, expanded graphite, commercial Graphene nanoplates and natural graphite, in a commercial epoxy matrix. The properties of dielectric and thermal conductivity and permeability to oxygen of the composites were studied and compared to those of the unfilled epoxy matrix.

[4] Presents a theoretical and experimental study on thermal conductivities of silica aerogel, xonotlite - type calcium silicate and xonotlite-aerogel composite insulation material. The thermal conductivities of the insulation material are measured from 300 to $970 \mathrm{~K}$ and from $0.045 \mathrm{~Pa}$ to atmospheric pressure.

[5] Obtained an analytical solution for two-dimensional transient heat conduction in fiber-reinforced multilayer cylindrical composites. Separation of variables method is employed to develop the transient temperature fields. The effect of the fibers' angle on the transient heat conduction behaviors is investigated.

[6] Developed a sandwich construction as a thermal management system, to have both superior thermal conductivity and structural integrity. The sandwich construction consists of a carbon foam core and unidirectional graphite/epoxy composite face sheets. An emphasis was put on enhancing the thermal conductivity of each phase of sandwich construction as well as interface between the phases. Property variation and anisotropy were observed with the highly conductive graphitic carbon foam. Co-curing of the composite face sheets with the carbon foam core was demonstrated to minimize the thickness of the adhesive layer between the face sheets and the core to produce the best 
construction of those tested. Comparison made with an adhesively bonded specimen shows that the co-curing is a more efficient method to enhance the through-thickness conductivity.

[7] The radiative properties and heat transfer in fiber-loaded silica aerogel composites were investigated. The randomly parameterized 2-D fiber distribution was generated to simulate a very realistic material structure. The finite volume method was used to solve a two flux radiation model and the steady-state energy equation to calculate the effective thermal conductivity of the composite.

[8] Studies the thermal radiative transfer in silica aerogel and silica aerogel composite insulation materials (a xonotliteaerogel composite and a ceramic fiber-aerogel composite). The radiative conductivity of each sample, deduced from the overall thermal conductivity measured using the transient hotstrip (THS) method.

In the present research the natural convection heat transfer is investigated in an inclined annulus used as heat exchanger. The material taken for the annulus was based on previous experimental work which used graphite/epoxy composite material. The ranges of the parameters affected on the study are modified Rayleigh number $\left(10 \leq \mathrm{Ra}^{*} \leq 500\right)$ and the annulus inclination angle $\delta(0,30,45,60$ and 90$)$. Two types of optimization will be performed in terms of effective thermal conductivity: minimization and maximization of thermal conductivity.

\section{MATHEMATICAL MODEL}

Fig.1. illustrates the schematic diagram of the geometry and the Cartesian coordinate system.

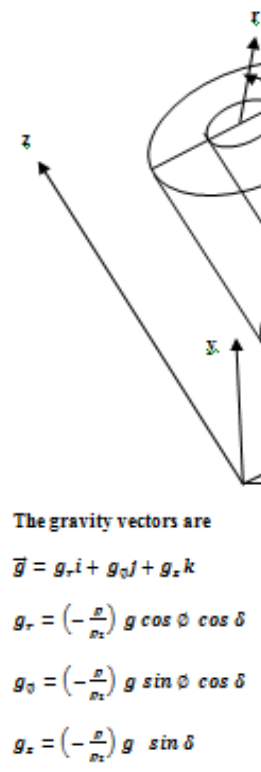

a. Coordinates system

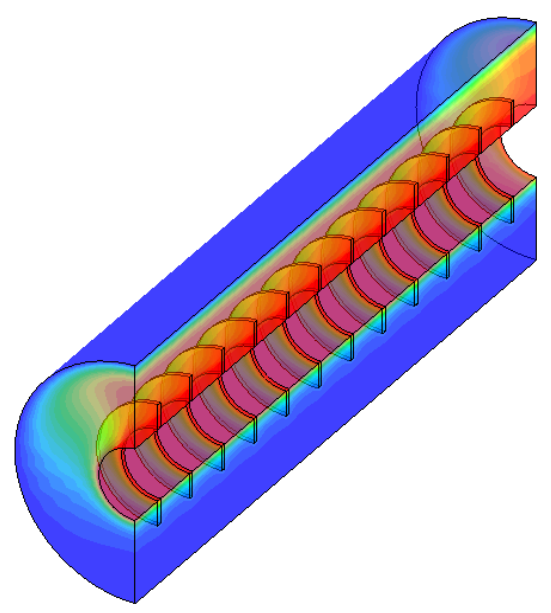

a. Geometry

Fig.1: Coordinates system and geometry

In order to model the incompressible flow in the porous medium, the steady-state equations of the Darcy flow model, namely, the mass, the momentum (Darcy), the energy conservation laws and the Boussinesq's approximation are employed. These equations in vectorial notation are given by [9].

\section{GOVERNING EQUATIONS}

The supplementary equation applied is:

$\rho=\rho_{2}\left[1-\beta\left(T-T_{2}\right)\right]$

Where:

$\beta=\frac{1}{\rho} \frac{\partial \rho}{\partial T}$

$\beta$ is the thermal coefficient of the volume expansion, this constant is evaluated at $T_{2}$ which is the temperature at the inner surface of the outer cylinder, $\rho_{2}$ is the density at $T_{2}$ and $\rho$ is the density at $\mathrm{T},[\mathbf{1 0}]$.

Darcy's law states that the volume average velocity through the porous material is proportional with the pressure gradient [11] and it will be used in this study. The governing equations are the continuity, momentum, energy and fin equations [12].

The vorticity vector $\Omega$ and a vector potential $\Psi$ with its components [13]:

$\Psi=\left(\psi_{r}, \psi_{\emptyset}, \psi_{z}\right)$

Defined by:

$U=\alpha_{e f f} \nabla \mathrm{X} \Psi$

$\nabla^{2} \psi_{r}=\frac{1}{R} \frac{\partial U_{Z}}{\partial \emptyset}-\frac{\partial U_{\emptyset}}{\partial Z}$

$\nabla^{2} \psi_{\varnothing}=\frac{\partial U_{r}}{\partial Z}-\frac{\partial U_{z}}{\partial R}$ 
$\nabla^{2} \psi_{z}=\frac{1}{R} \frac{\partial\left(R U_{\emptyset}\right)}{\partial R}-\frac{1}{R} \frac{\partial U_{r}}{\partial \emptyset}$

\section{NON DIMENSIONAL VARIABLES}

The characteristic length used is $r_{2}$ [10] to convert the governing equations to the dimensionless form, the dimensionless magnitudes defined as follow [14]:

$R=\frac{r}{r_{2}}, \quad Z=\frac{z}{r_{2}}, \quad U_{r}=\frac{u_{r} l}{\alpha_{e f f}}, \quad U_{\emptyset}=\frac{u_{\varnothing} l}{\alpha_{e f f}}, \quad U_{z}=\frac{u_{z} l}{\alpha_{e f f}}$

$\theta=\left(T-T_{2}\right) /\left(T_{1}-T_{2}\right), \quad P=\frac{p K l}{\alpha_{e f f} \mu_{f} r_{2}}$

$R_{a}^{*}=g \beta \frac{K\left(T_{1}-T_{2}\right)\left(r_{1}-r_{2}\right)}{\alpha_{e f f} \vartheta}$

For fins (see Fig. 2) $\quad S_{1}=\frac{S}{2 r_{2}}, S_{2}=\frac{\frac{S}{2}+t}{r_{2}}, \quad H_{1}=\frac{H_{f}}{r_{2}}$

Substitute these dimensionless magnitudes in the governing equations and taking curl of the momentum equations to eliminate pressure terms, the momentum equations will be:

$R_{a}^{*} \frac{l}{\left(r_{1}-r_{2}\right)}\left[\frac{1}{R} \sin \delta \frac{\partial \theta}{\partial \emptyset}+\sin \emptyset \cos \delta \frac{\partial \theta}{\partial z}\right]=-\frac{\partial^{2} \psi_{r}}{\partial R^{2}}-\frac{1}{R^{2}} \frac{\partial\left(R \psi_{r}\right)}{\partial R}-$ $\frac{2}{R} \frac{\partial \psi_{r}}{\partial R}-\frac{1}{R^{2}} \frac{\partial^{2} \psi_{r}}{\partial \emptyset^{2}}-\frac{\partial^{2} \psi_{r}}{\partial Z^{2}}-\frac{2}{R} \frac{\partial \psi_{z}}{\partial Z}$

$R_{a}^{*} \frac{l}{\left(r_{1}-r_{2}\right)}\left[\cos \emptyset \cos \delta \frac{\partial \theta}{\partial Z}-\sin \delta \frac{\partial \theta}{\partial R}\right]=-\frac{\partial^{2} \psi_{\emptyset}}{\partial Z^{2}}-\frac{1}{R^{2}} \frac{\partial^{2} \psi_{\emptyset}}{\partial \emptyset^{2}}-$ $\frac{\partial^{2} \psi_{\emptyset}}{\partial R^{2}}-\frac{2}{R^{2}} \frac{\partial \psi_{r}}{\partial \emptyset}+\frac{\psi_{\emptyset}}{R^{2}}-\frac{1}{R} \frac{\partial \psi_{\emptyset}}{\partial R}$

$R_{a}^{*} \frac{l}{\left(r_{1}-r_{2}\right)} \cos \delta\left[\frac{1}{R} \cos \emptyset \frac{\partial \theta}{\partial \emptyset}+\sin \emptyset \frac{\partial \theta}{\partial R}\right]=-\frac{\partial^{2} \psi_{z}}{\partial R^{2}}-\frac{1}{R} \frac{\partial \psi_{z}}{\partial R}-$

$\frac{1}{R^{2}} \frac{\partial^{2} \psi_{z}}{\partial \emptyset^{2}}-\frac{\partial^{2} \psi_{z}}{\partial Z^{2}}$

The vector potential equation was obtained in the dimensionless form as [14]:

$\nabla^{2} \psi_{r}=-\frac{\partial^{2} \psi_{r}}{\partial R^{2}}-\frac{1}{R^{2}} \frac{\partial\left(R \psi_{r}\right)}{\partial R}-\frac{2}{R} \frac{\partial \psi_{r}}{\partial R}-\frac{1}{R^{2}} \frac{\partial^{2} \psi_{r}}{\partial \emptyset^{2}}-\frac{\partial^{2} \psi_{r}}{\partial Z^{2}}-\frac{2}{R} \frac{\partial \psi_{z}}{\partial Z}$

$\nabla^{2} \psi_{\emptyset}=-\frac{\partial^{2} \psi_{\emptyset}}{\partial Z^{2}}-\frac{1}{R^{2}} \frac{\partial^{2} \psi_{\emptyset}}{\partial \emptyset^{2}}-\frac{\partial^{2} \psi_{\emptyset}}{\partial R^{2}}-\frac{2}{R^{2}} \frac{\partial \psi_{r}}{\partial \emptyset}+\frac{\psi_{\emptyset}}{R^{2}}-\frac{1}{R} \frac{\partial \psi_{\emptyset}}{\partial R}$

$\nabla^{2} \psi_{z}==-\frac{\partial^{2} \psi_{z}}{\partial R^{2}}-\frac{1}{R} \frac{\partial \psi_{z}}{\partial R}-\frac{1}{R^{2}} \frac{\partial^{2} \psi_{z}}{\partial \emptyset^{2}}-\frac{\partial^{2} \psi_{z}}{\partial Z^{2}}$

And the energy equation will be:

$$
\begin{aligned}
& \left(\frac{1}{R} \frac{\partial \psi_{z}}{\partial \emptyset}-\frac{\partial \psi_{\emptyset}}{\partial Z}\right) \frac{\partial \theta}{\partial R}+\frac{1}{R}\left(\frac{\partial \psi_{r}}{\partial Z}-\frac{\partial \psi_{z}}{\partial R}\right) \frac{\partial \theta}{\partial \emptyset}+\left(\frac{\psi_{\emptyset}}{R}+\frac{\partial \psi_{\emptyset}}{\partial R}-\right. \\
& \left.\frac{1}{R} \frac{\partial \psi_{r}}{\partial \emptyset}\right) \frac{\partial \theta}{\partial Z}=\frac{l}{r_{1}}\left(\frac{\partial^{2} \theta}{\partial R^{2}}+\frac{1}{R} \frac{\partial \theta}{\partial R}+\frac{1}{R^{2}} \frac{\partial^{2} \theta}{\partial \emptyset^{2}}+\frac{\partial^{2} \theta}{\partial Z^{2}}\right)
\end{aligned}
$$

And fin equation will be:

$\frac{\partial \theta}{\partial R}+\frac{\theta}{R}+\frac{1}{R} \frac{\partial \theta}{\partial \emptyset}+\frac{\partial \theta}{\partial Z}=0$

\subsection{Dimensionless Hydraulic Boundary Conditions}

For the vector potential field, the boundary conditions are given as:
$\frac{1}{R} \frac{\partial\left(R \psi_{r}\right)}{\partial R}=\psi_{\emptyset}=\psi_{z}=0$
at $R=R_{1}, 1$
$\psi_{r}=\frac{\partial \psi_{\emptyset}}{\partial \emptyset}=\psi_{z}=0$
at $\varnothing=0, \pi$
$\psi_{r}=\psi_{\emptyset}=\frac{\partial \psi_{z}}{\partial Z}=0$
at $\mathrm{Z}=0, \mathrm{~L}$

The boundary conditions for fins are given as:

$\frac{1}{R} \frac{\partial\left(R \psi_{r}\right)}{\partial R}=\frac{\partial \psi_{\emptyset}}{\partial \emptyset}=\frac{\partial \psi_{z}}{\partial Z}=0$

On the fin faces which were located on the following planes

(Fin base)

At $\mathrm{R}=R_{l} \quad$ for $\quad \phi=0, \pi$

(Fin tip)

At $r=r_{l}+H_{f} \quad$ for $\quad \phi=0, \pi$
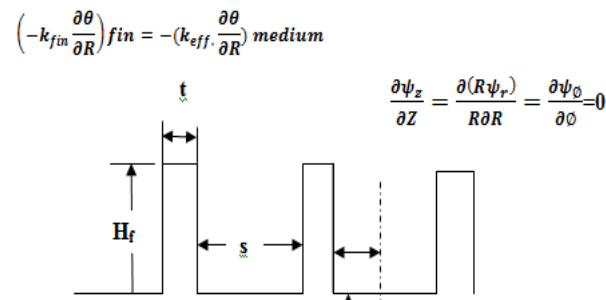

$\left.\frac{\partial \theta}{\partial Z}\right)$ fin $=-\left(k_{\text {eff }} . \partial \theta / \partial Z\right)$ medium

$\left(-k_{-}\right.$fin $\left.\partial \theta / \partial \emptyset\right)$ fin $=-\left(k_{\text {eff }}, \partial \theta / \partial \emptyset\right)$ medium

At $S_{1}$ and $S_{2}$ at any $r$ and $\varnothing$

Fig. 2: fin boundary conditions

\subsection{Dimensionless Thermal Boundary}

Conditions

For the temperature field, the dimensionless thermal boundary conditions are:

$$
\begin{aligned}
& \theta=1 \quad \text { at } R=R_{1}=\frac{r_{1}}{r_{2}} \\
& \theta=0 \quad \text { at } R=R_{2}=1
\end{aligned}
$$




$$
\begin{aligned}
& \frac{\partial \theta}{\partial \emptyset}=0 \quad \text { at } \emptyset=0, \pi \\
& \frac{\partial \theta}{\partial Z}=0 \quad \text { at } Z=0, L
\end{aligned}
$$$$
\text { At } \quad \mathrm{R}=\mathrm{H}
$$$$
-\left.k_{f i n} \frac{\partial \theta}{\partial R}\right|_{f i n}=-\left.k_{\text {eff }} \frac{\partial \theta}{\partial R}\right|_{\text {medium }}
$$

$$
\begin{aligned}
& \text { At } S_{1} \text { and any } R \text { and } \varnothing \\
& \text { At } \mathrm{S}_{2} \text { and any } \mathrm{R} \text { and } \varnothing
\end{aligned}
$$$$
-\left.k_{f i n} \frac{\partial \theta}{\partial Z}\right|_{\text {fin }}=-\left.k_{\text {eff }} \frac{\partial \theta}{\partial Z}\right|_{\text {medium }}
$$$$
\text { At } \varnothing=0, \pi \quad \text { and any } \mathrm{R}
$$$$
-\left.k_{f i n} \frac{\partial \theta}{\partial \emptyset}\right|_{f i n}=-\left.k_{\text {eff }} \frac{\partial \theta}{\partial \emptyset}\right|_{\text {medium }}
$$

$k_{e f f}=(1-\varepsilon) k_{s}+\varepsilon k_{f}$

The conduction heat transfer can also be affected by the fiber orientation. When the axis of the fiber is perpendicular to heat flux, the conduction heat transfer would get the minimum value. When the axis of the fiber is parallel to heat flux, the thermal conductivity would be the largest.

Graphite fibers and epoxy have conductive heat transfer coefficients of 14.74 and $0.19 \mathrm{~W} / \mathrm{m} \mathrm{K}$, respectively. The conductive coefficients of the graphite fibers and the epoxy matrix differ greatly. The thermal conductivity of this composite laminate parallel to the fibers is much greater than that perpendicular to the fibers. Graphite/epoxy composite material has a thermal conductivity in parallel direction of fibers of $11.1 \mathrm{~W} / \mathrm{m} \mathrm{K}$ and in perpendicular direction of fibers of $0.87 \mathrm{~W} / \mathrm{m} \mathrm{K}$ and with volumetric percentage of fibers of 75 [15].

\section{COMPUTATIONAL TECHNIQUE}

Eq. (7, 8, 9, 13 and 14) were transformed into the finite difference equations, where the upwind differential method in the left hand side of the energy eq.(13) and the centered space differential method for the other terms were used, and solved by using (SOR) method [11]. A computer program was built using MATLAB-7 to get the results of the problem.

The convergence criterion is taken by inequality:

$\operatorname{Max} .\left|\frac{\theta^{n+1}-\theta^{n}}{\theta^{n}}\right| \leq 10^{-8}$
The number of grid points used was 21 grid points in the $\mathrm{R}-$ direction, 31 in the $\phi$-direction and 301 in the $\mathrm{Z}$ - direction which seems reasonable and will be used in the present study.

\subsection{Calculation of Local and Average Nusselt Number}

Local Nusselt number can be obtained as follows [10]:

$N u=\frac{q\left(r_{2}-r_{1}\right)}{k\left(T_{1}-T_{2}\right)}$

The local Nusselt number $N u_{1}$ and $N u_{2}$ on the inner and the outer cylinders are as follow [10]:

$N u_{1}=-\left(1-R_{1}\right)\left(\frac{\partial \theta}{\partial R}\right)_{R=R_{1}}$

$N u_{2}=-\left(1-R_{1}\right)\left(\frac{\partial \theta}{\partial R}\right)_{R=1}$

The average Nusselt number $N u_{\text {in }}$ and $N u_{\text {out }}$ on the inner and the outer cylinders are defined as:

$N u_{\text {in }}=-\left(1-R_{1}\right) \frac{1}{\pi L} \int_{0}^{L} \int_{0}^{\pi}\left(\frac{\partial \theta}{\partial R}\right)_{R=R_{1}} d \emptyset d Z$

$N u_{\text {out }}=-\left(1-R_{1}\right) \frac{1}{\pi L} \int_{0}^{L} \int_{0}^{\pi}\left(\frac{\partial \theta}{\partial R}\right)_{R=1} d \emptyset d Z$

\section{RESULTS AND DISCUSSION}

\subsection{Isotherms and Streamlines Field}

The dimensionless temperature distribution and streamlines within the enclosure are presented in a contour maps form in the $(\mathrm{R}-\phi)$ plane.

Fig.3 to Fig. 16 show the isotherms and streamlines for the two cases of maximum and minimum thermal conductivity of the Graphite fibers/epoxy and for different values of $\mathrm{Ra}$ and annulus inclination angle. In Fig. 3 the symmetry of the temperature distribution for horizontal annulus is clear and it was observed that for $\delta=90^{\circ}$ in Fig. 4 the effect of the fins will be clear and the meanders in temperature distribution will occur. The streamlines have high intensity at the boundaries and less values in the regions away from the boundary which take beans form and expand as $\delta$ becomes $90^{\circ}$ (vertical annulus). Increase $\mathrm{Ra}^{*}$ for $\delta=0^{\circ}$ results in a thicker cold layer near the bottom wall and a high temperature field near the top wall. More heat is transported upward, and a large difference of temperature is observed between the upper and lower parts of the annulus as shown in

Fig. 5. The streamlines have high intensity in the boundaries and at the bottom and as $\delta$ increase to $30^{\circ}, 45^{\circ}, 60^{\circ}$ and $90^{\circ}$ the center of the streamlines of negative values expand and fade away to the lower region. 


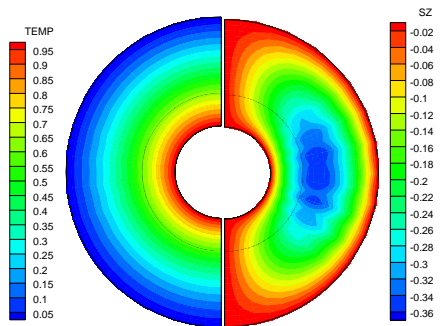

Fig. 3: Isothermal and streamlines contours for $\mathrm{Ra}^{*}=10$, $\delta=0, \mathrm{k}=11.1 \mathrm{~W} / \mathrm{m} \mathrm{K}$

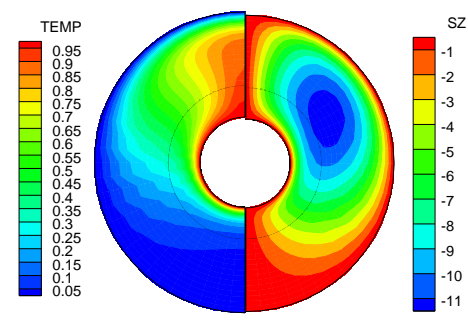

Fig. 4: Isothermal and streamlines contours for $\mathrm{Ra}^{*}=10$, $\delta=90, \mathrm{k}=11.1 \mathrm{~W} / \mathrm{m} \mathrm{K}$

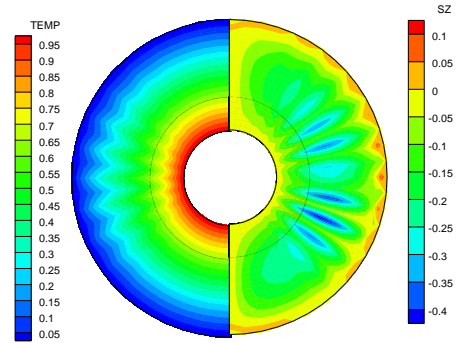

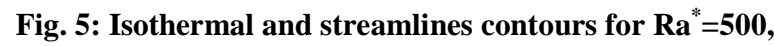
$\delta=0, \mathrm{k}=11.1 \mathrm{~W} / \mathrm{m} \mathrm{K}$

The isotherms in the upper region shift towards the outer (cold) cylinder and the cold lower region will dwindle as shown in Fig.6 to Fig. 9 and a swell of the isothermal lines can observed when $\mathrm{Ra}^{*}$ increase which implies a low $\mathrm{Nu}$ on the inner cylinder and a high $\mathrm{Nu}$ on the outer cylinder.

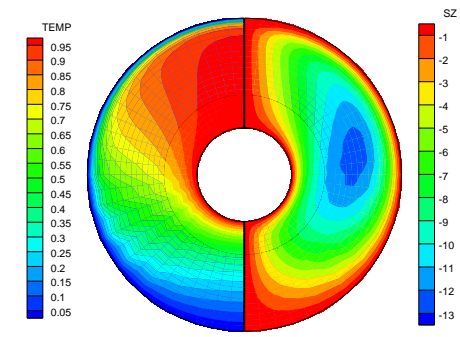

Fig. 6: Isothermal and streamlines contours for $\mathbf{R a}^{*}=500$, $\delta=30, \mathrm{k}=11.1 \mathrm{~W} / \mathrm{m} \mathrm{K}$

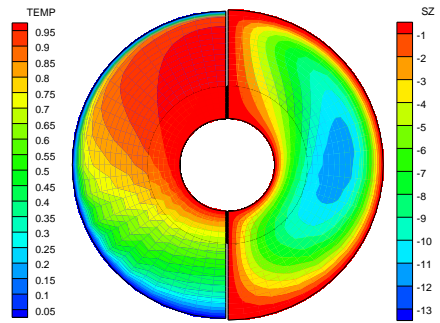

Fig. 7: Isothermal and streamlines contours for $\mathbf{R a}^{*}=\mathbf{5 0 0}$,

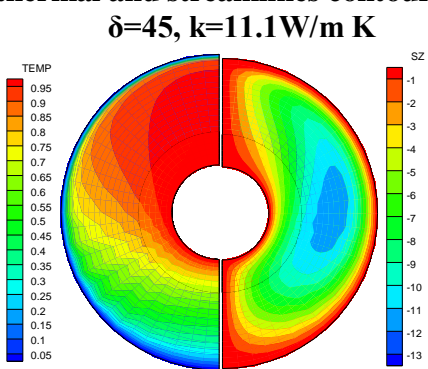

Fig. 8: Isothermal and streamlines contours for $\mathbf{R a}^{*}=\mathbf{5 0 0}$, $\delta=60, k=11.1 \mathrm{~W} / \mathrm{m} \mathrm{K}$

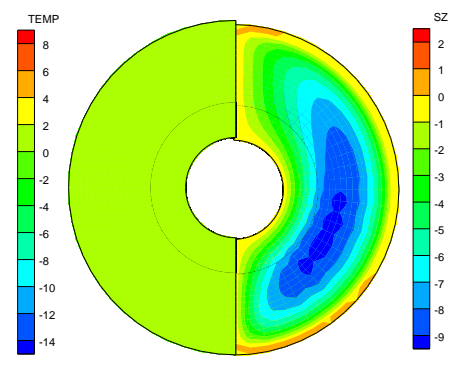

Fig. 9: Isothermal and streamlines contours for $\mathbf{R a}^{*}=500$, $\delta=90, \mathrm{k}=11.1 \mathrm{~W} / \mathrm{m} \mathrm{K}$

Fig.10 to Fig. 16 show the same behave for isotherms and streamlines as previous figures but of course with less heat transfer, so for $\mathrm{k}=0.87 \mathrm{~W} / \mathrm{m} \mathrm{K}$ (perpendicular direction of fibers), the region in the bottom is colder than that for the case of $\mathrm{k}=11.1 \mathrm{~W} / \mathrm{m} \mathrm{K}$ (parallel direction of fibers) for the same values of $\mathrm{Ra}^{*}$ and $\delta$. Since this research was achieved for a steady state laminar region, thus the warm region at the top end when $\delta=90^{\circ}$ come to be as a concentric circles located at the center of the annulus and distributed between the hot and cold cylinders Fig. 16. 


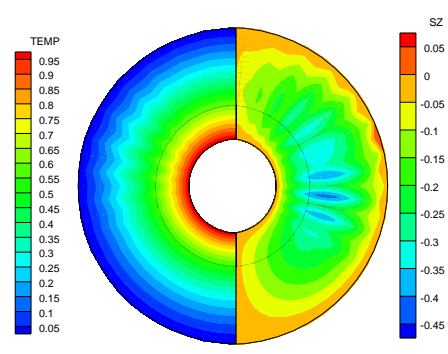

Fig. 10: Isothermal and streamlines contours for $\operatorname{Ra}^{*}=10, \delta=0, k=0.87 \mathrm{~W} / \mathrm{m} \mathrm{K}$

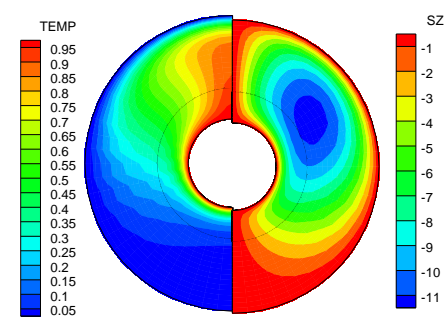

Fig. 11: Isothermal and streamlines contours for $\mathbf{R a}^{*}=\mathbf{5 0 0}$, $\delta=0, k=0.87 \mathrm{~W} / \mathrm{m} \mathrm{K}$

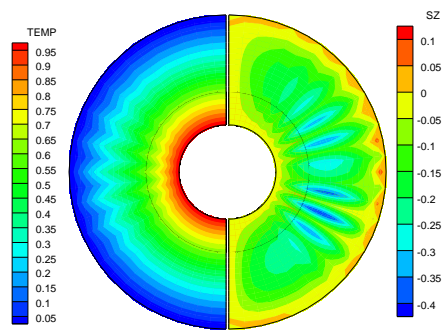

Fig. 12: Isothermal and streamlines contours for $\mathbf{R a}^{*}=\mathbf{1 0}$, $\delta=90, k=0.87 \mathrm{~W} / \mathrm{m} \mathrm{K}$

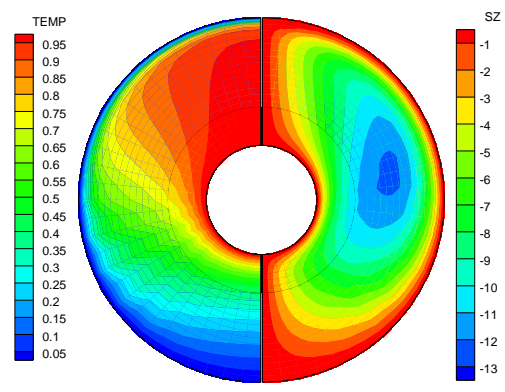

Fig. 13: Isothermal and streamlines contours for $\mathbf{R a}^{*}=500$, $\delta=30, k=0.87 \mathrm{~W} / \mathrm{m} \mathrm{K}$

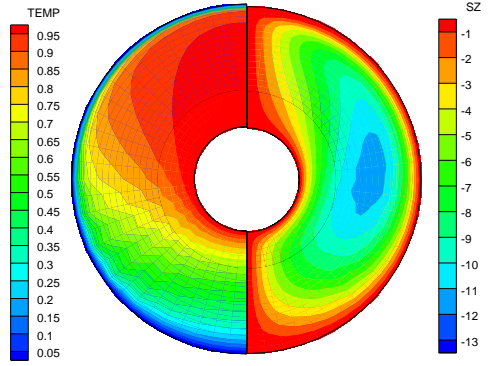

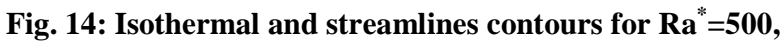
$\delta=45, k=0.87 \mathrm{~W} / \mathrm{m} \mathrm{K}$

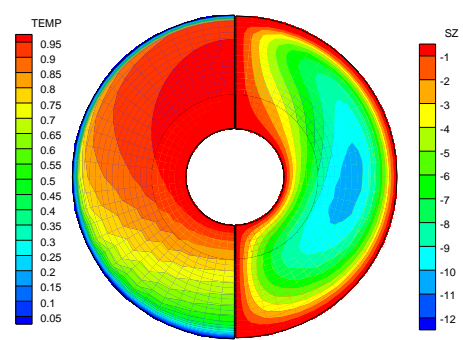

Fig. 15: Isothermal and streamlines contours for $\mathbf{R a}^{*}=\mathbf{5 0 0}$, $\delta=60, k=0.87 \mathrm{~W} / \mathrm{m} \mathrm{K}$

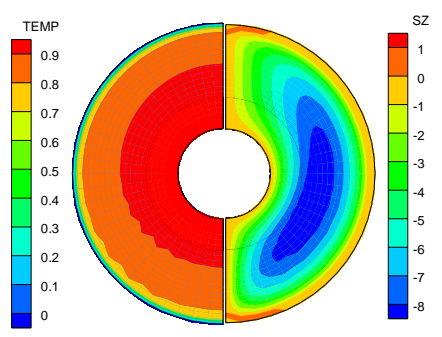

Fig. 16: Isothermal and streamlines contours for $\mathbf{R a}={ }^{*} \mathbf{5 0 0}$, $\delta=90, \mathrm{k}=0.87 \mathrm{~W} / \mathrm{m} \mathrm{K}$

\subsection{Average and Local Nusselt Numbers}

Fig.17 and Fig. 18 show the variation in the average Nusselt number on the outer cold cylinder with the inclination angle for different values of $\mathrm{Ra}^{*}$ and for the two cases of minimization and maximization of thermal conductivity. It is clear that $\mathrm{Nu}$ decrease with the increase of $\delta$ and increase with the increase of $\mathrm{Ra}^{*}$. The deviation between the average $\mathrm{Nu}$ in the two cases is clear in Fig.17 and Fig. 18 which is equal to $5.1 \%$ for $\delta=0^{\circ}$ and $10 \%$ for $\delta=90^{\circ}$. For the inner hot cylinder the deviation between the two cases is not significant for $\delta=0^{\circ}$ but it will be significant only for vertical annulus at $\mathrm{Ra}^{*}=500$ and equal to $12.8 \%$. 


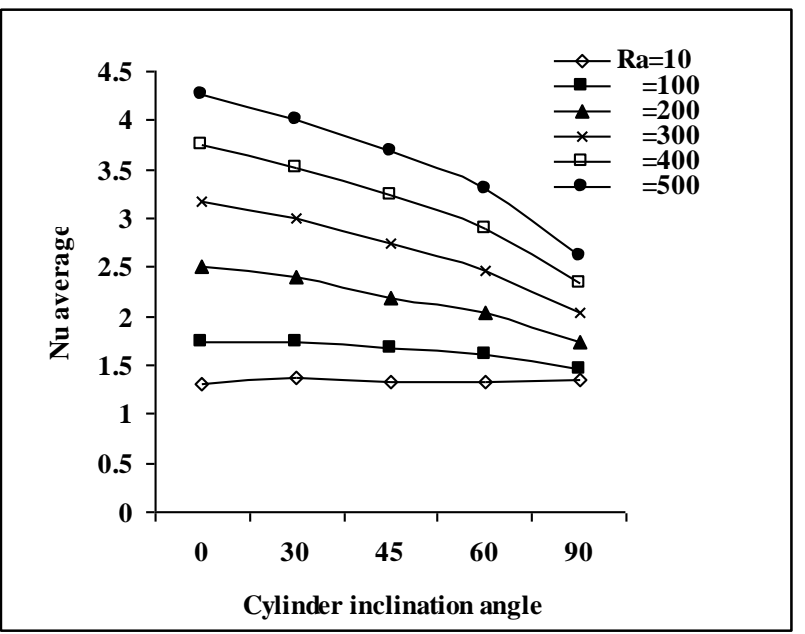

Fig.17: Variation of $\mathrm{Nu}_{\text {average }}$ with the inclination angle on the outer cold cylinder for $k=0.87 \mathrm{~W} / \mathrm{m} \mathrm{K}$

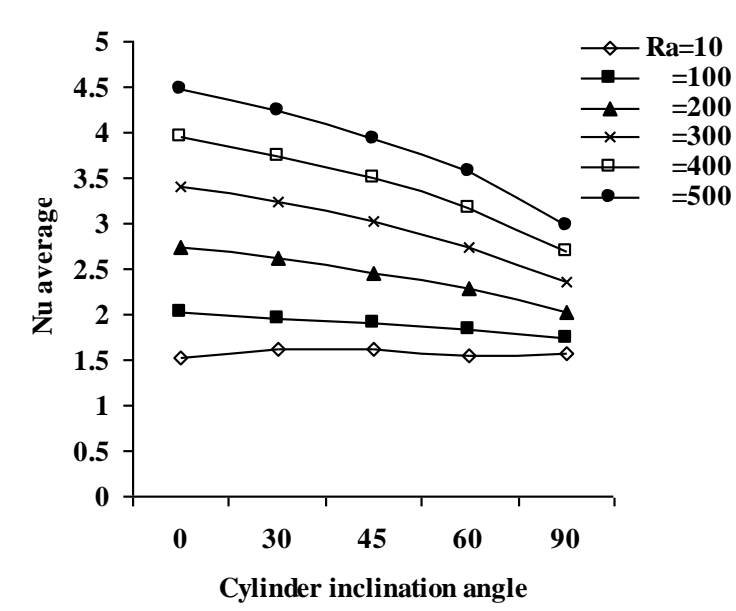

Fig.18: Variation of $\mathrm{Nu}_{\text {average }}$ with the inclination angle on the outer cold cylinder for $\mathrm{k}=11.1 \mathrm{~W} / \mathrm{m} \mathrm{K}$

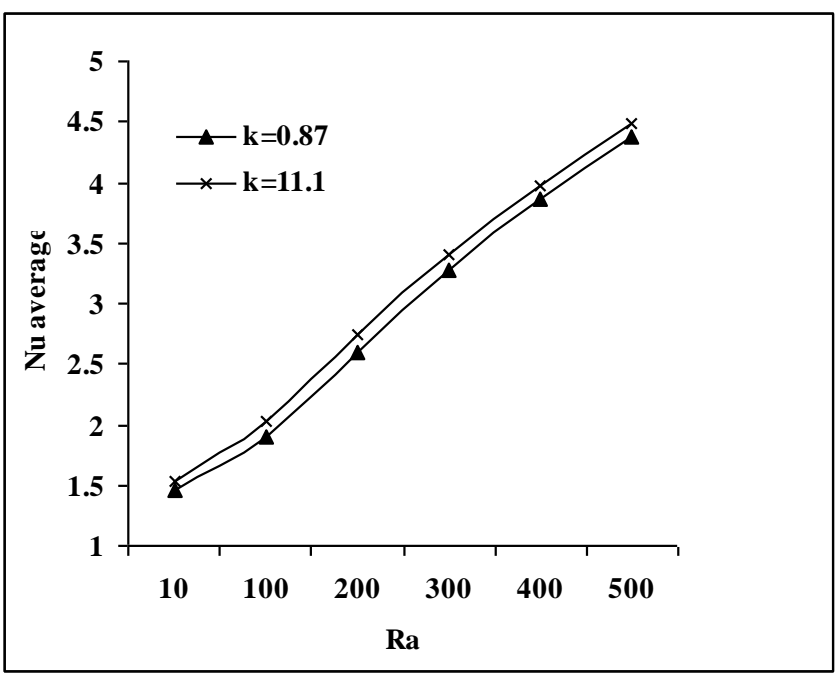

Fig.19: Variation of $\mathrm{Nu}_{\text {average }}$ with $\mathrm{Ra}$ on the outer cold cylinder for $\delta=0^{\circ}$

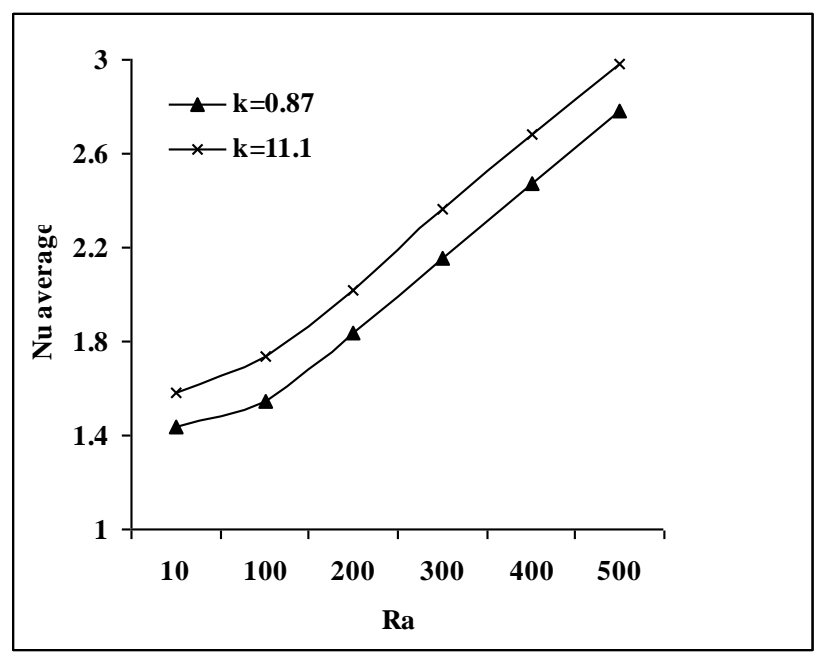

Fig.20: Variation of $\mathrm{Nu}_{\text {average }}$ with $\mathrm{Ra}$ on the outer cold cylinder for $\delta=90^{\circ}$

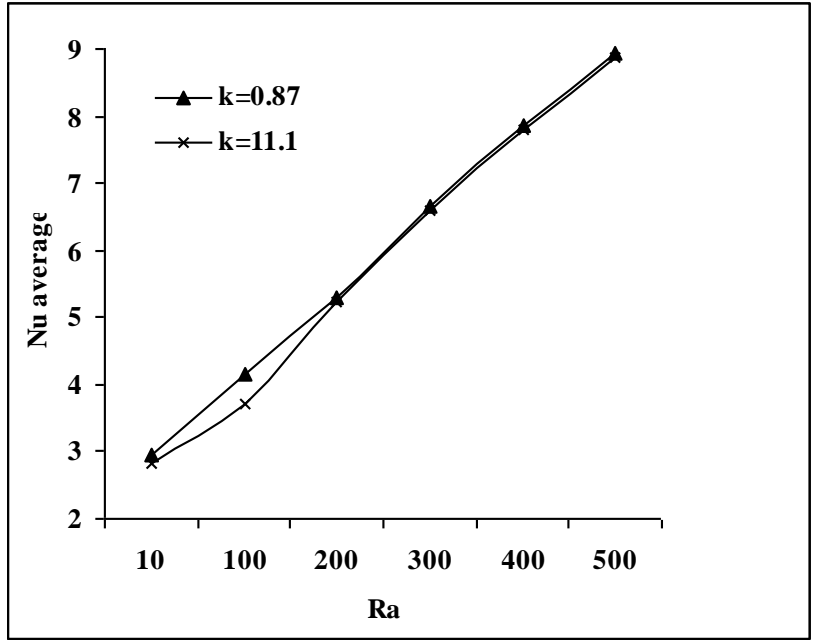

Fig.21: Variation of $\mathrm{Nu}_{\text {average }}$ with $\mathrm{Ra}$ on the inner hot cylinder for $\delta=0^{\circ}$

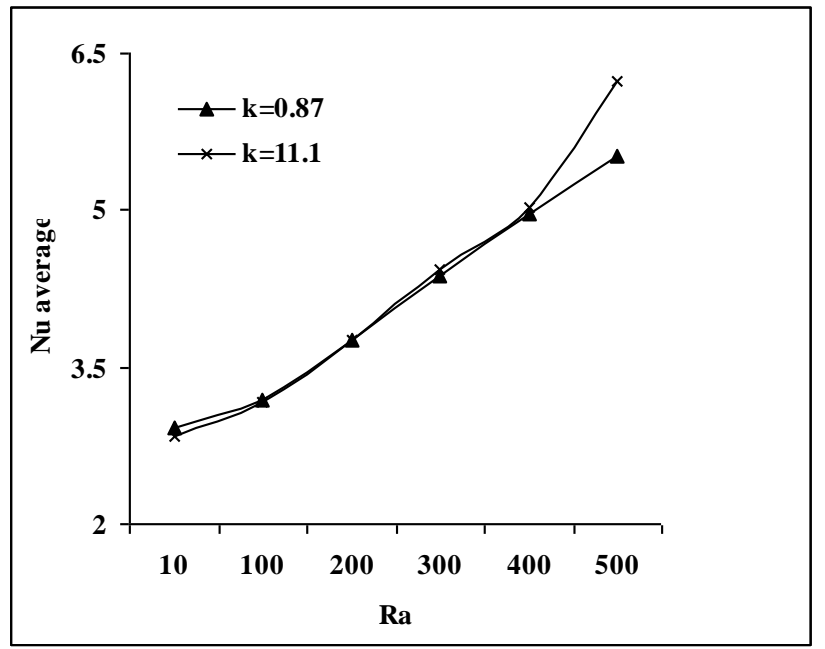

Fig.22: Variation of $\mathrm{Nu}_{\text {average }}$ with $\mathrm{Ra}$ on the inner hot cylinder for $\delta=90^{\circ}$ 
Distribution of local Nusselt number along the circumstance of the cold and hot cylinders is illustrated in Fig. 23 and Fig. 24 respectively at three locations; located at the top of the cylinder, at the center of the cylinder and the third at the bottom of the cylinder. These curves illustrate the cases for different values of parameters.

The local Nusselt number on the cold wall had an increasing trend and there were three regions which could be distinguished. The first region, where the local Nusselt number is relatively constant since the inner boundary layer of relative uniform thickness and give a little variation in local Nusselt number distribution.

The second region, where the local Nusselt number loosed the uniformity and a large gradient in its distribution could be observed and this is because the inner boundary layer got thicker in this region since heat removal by fluid decrease as the fluid ascend up.

The third region, where the local Nusselt number approached to its maximum values this was due to the formation of plume in this region. Formation of the plume occurred where the two convective currents coming from the two annulus halves, impinging with each other and moving together upward without mixing, leaving a relatively stagnant region under impinging point. This behavior is roughly similar for most of modified Rayleigh number whereas the behavior of the hot wall is to decrease.

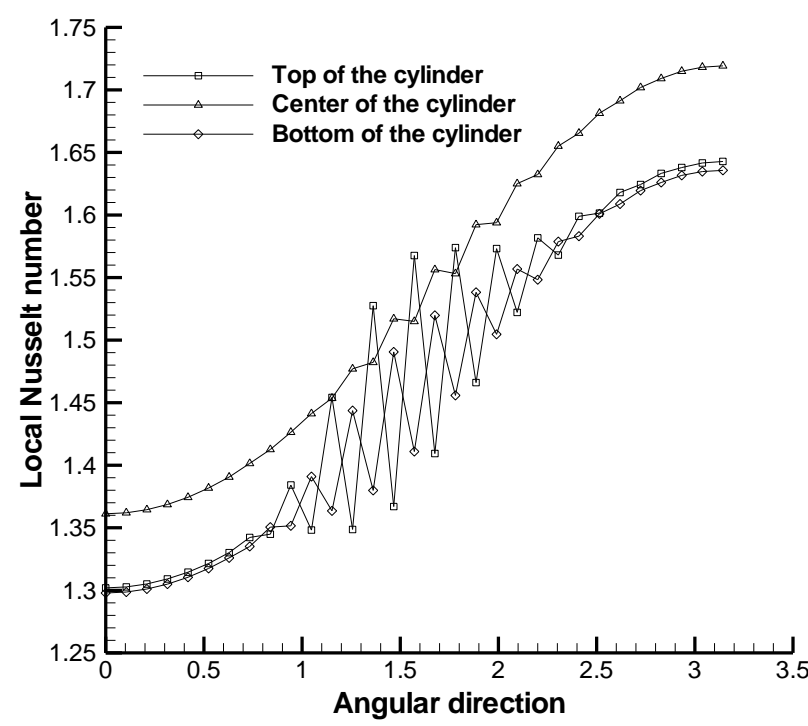

Fig.23: Variation of $\mathrm{Nu}_{\text {Local }}$ in angular direction on the cold cylinder for $R a=10, k=11.1 \mathrm{~W} / \mathrm{m} \mathrm{K} \delta=0^{\circ}$

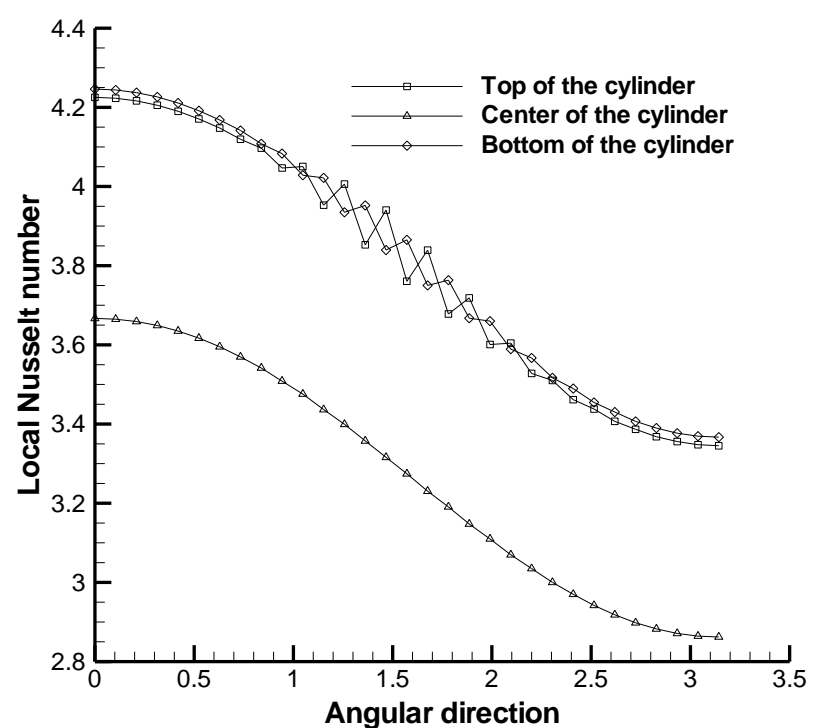

Fig.24: Variation of $\mathrm{Nu}_{\text {Local }}$ in angular direction on the hot cylinder for $\mathrm{Ra}=10, \mathrm{k}=11.1 \mathrm{~W} / \mathrm{m} \mathrm{K} \delta=0^{\circ}$

As shown in Fig. 25 and Fig. 26 respectively, the maximum value of the local Nusselt number at vertical position $\left(\delta=90^{\circ}\right)$ of the annulus. At high Ra* in Fig. 27 to Fig. 30 the local Nusselt number was high owing to the effect of convection mode of heat transfer, at low $\mathrm{Ra}^{*}$ the mode of heat transfer is conduction and its value increase with the increase of $\mathrm{Ra}^{*}$ and it is clear that the second region will be vanish or in other words the variation trend to be uniform.

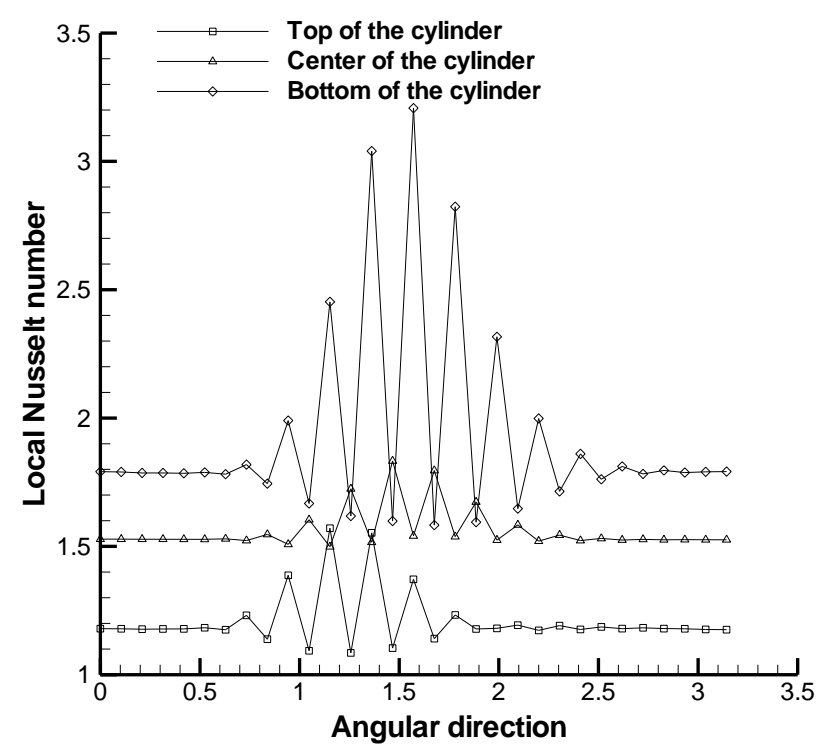

Fig.25: Variation of $\mathrm{Nu}_{\text {Local }}$ in angular direction on the cold cylinder for $R a=10, k=11.1 \mathrm{~W} / \mathrm{m} \mathrm{K} \delta=90^{\circ}$ 


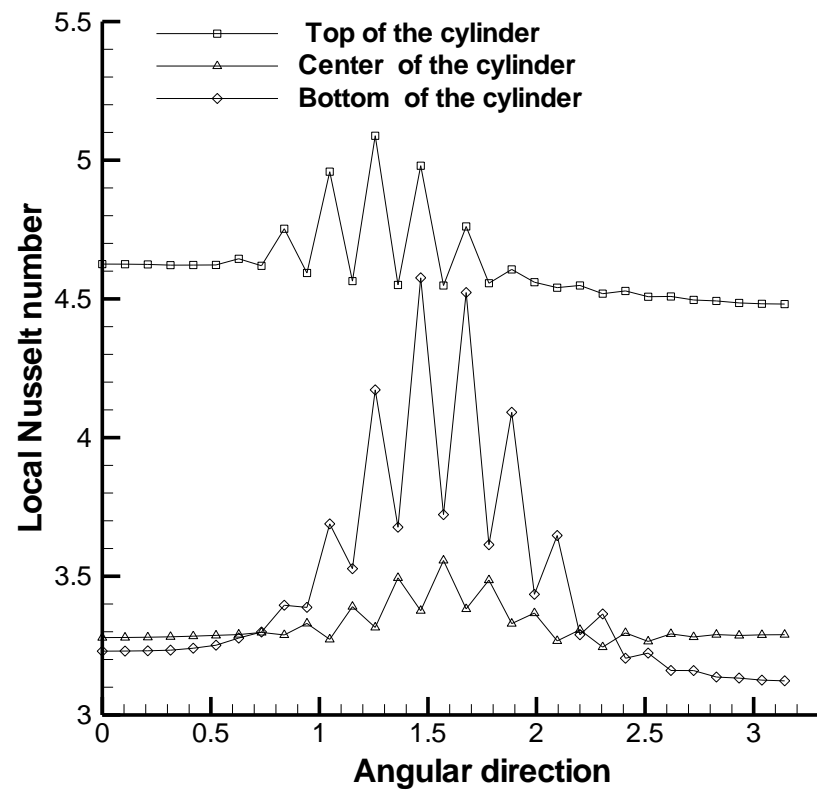

Fig.26: Variation of $\mathrm{Nu}_{\text {Local }}$ in angular direction on the hot cylinder for $\operatorname{Ra}=10, k=11.1 \mathrm{~W} / \mathrm{m} \mathrm{K} \delta=90^{\circ}$

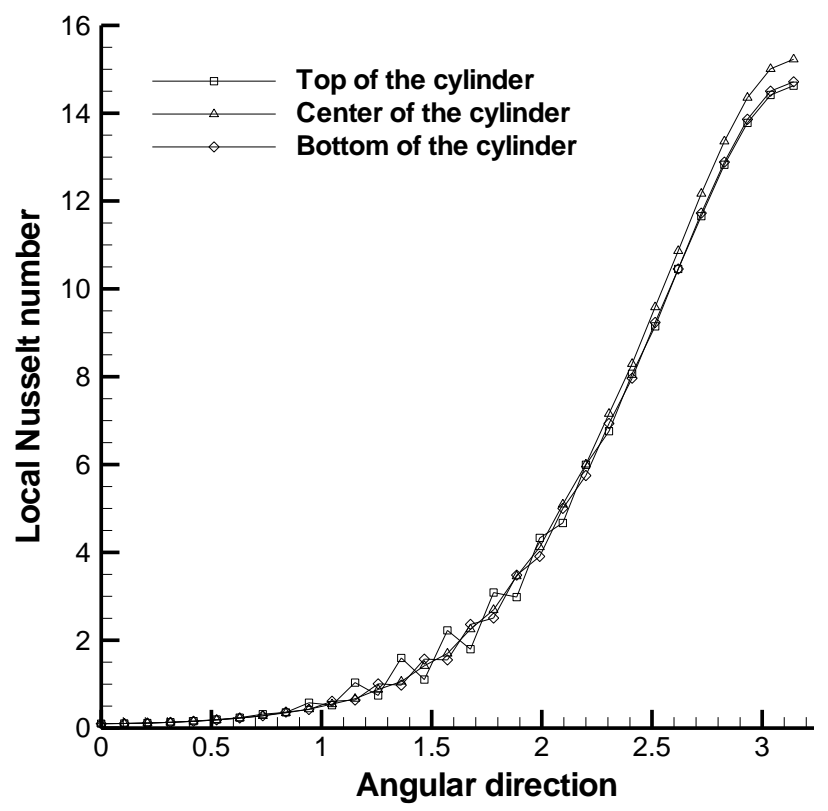

Fig.27: Variation of $\mathrm{Nu}_{\text {Local }}$ in angular direction on the cold cylinder for $\mathrm{Ra}=500, \mathrm{k}=11.1 \mathrm{~W} / \mathrm{m} \mathrm{K} \delta=0^{\circ}$

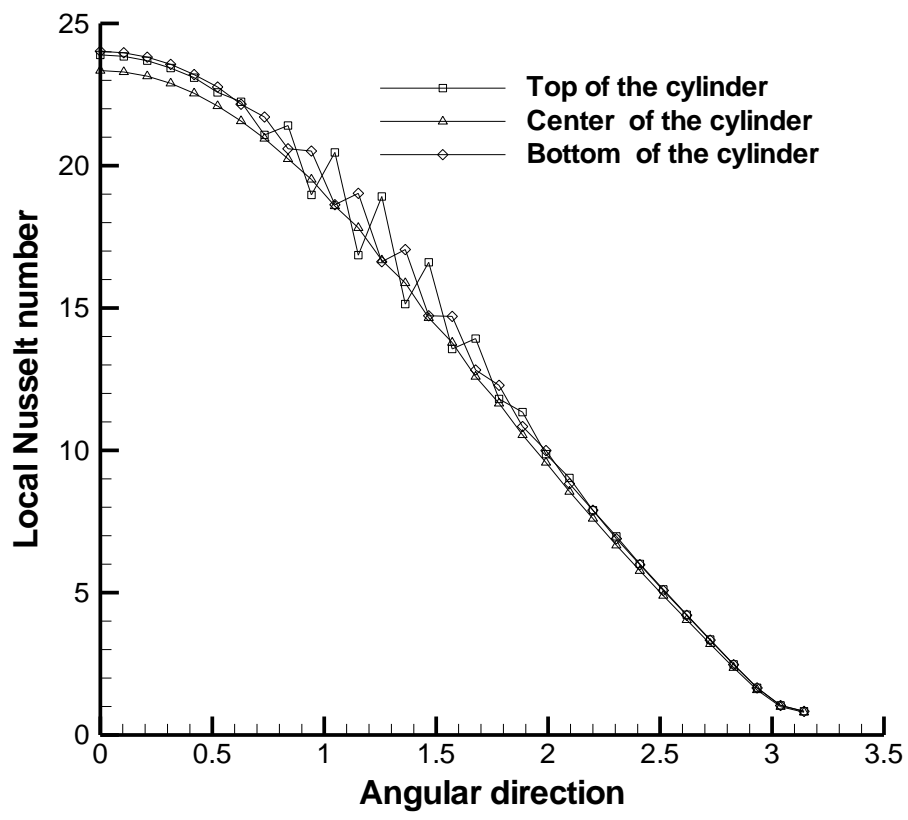

Fig.28: Variation of $\mathrm{Nu}_{\text {Local }}$ in angular direction on the hot cylinder for $\mathrm{Ra}=500, \mathrm{k}=11.1 \mathrm{~W} / \mathrm{m} \mathrm{K} \delta=0^{\circ}$

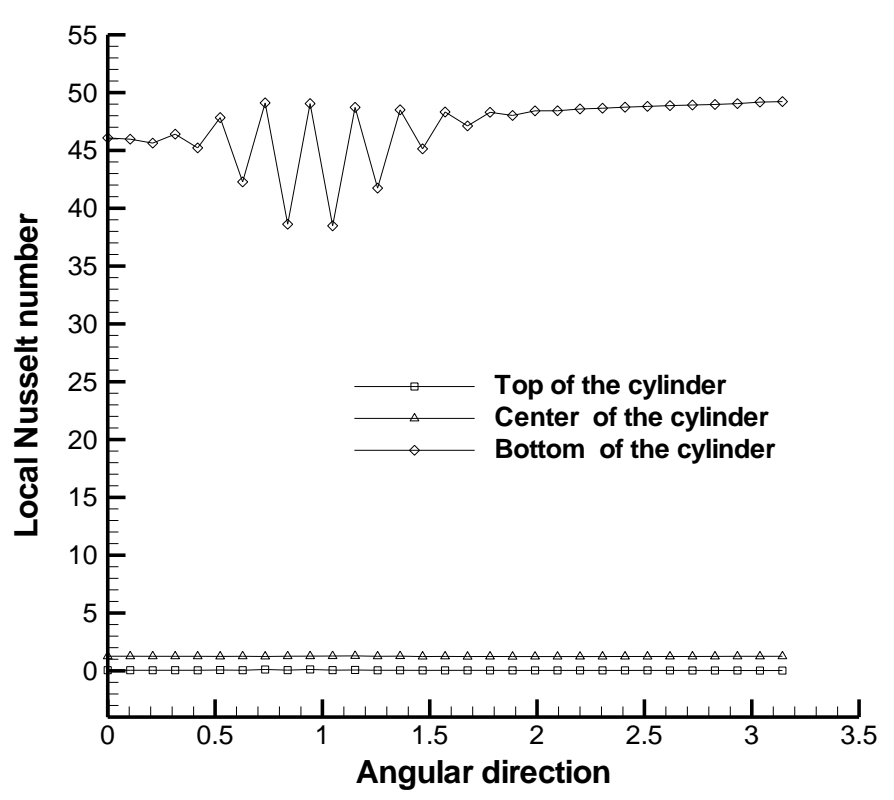

Fig.29: Variation of $\mathrm{Nu}_{\text {Local }}$ in angular direction on the cold cylinder for $\mathrm{Ra}=500, \mathrm{k}=11.1 \mathrm{~W} / \mathrm{m} \mathrm{K} \delta=90^{\circ}$ 


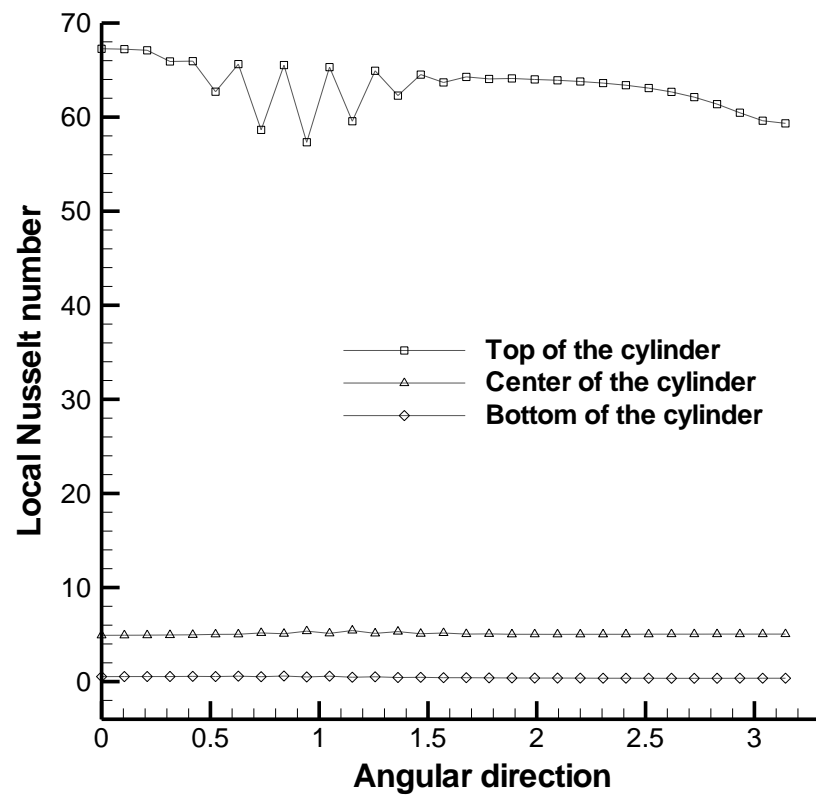

Fig.30: Variation of $\mathrm{Nu}_{\text {Local }}$ in angular direction on the hot cylinder for $\mathrm{Ra}=500, \mathrm{k}=11.1 \mathrm{~W} / \mathrm{m} \mathrm{K} \delta=90^{\circ}$

Fig. 31 and Fig. 32 for $\mathrm{k}=0.87 \mathrm{~W} / \mathrm{m} \mathrm{K}$ and $\delta=0^{\circ}$, there is only two regions and the non uniformity of the local Nusselt number will be significant. For other values of $\delta$ or $\mathrm{Ra}^{*}$ the behave is the same as the previous figures as shown in Fig. $\mathbf{3 3}$ and Fig. 38

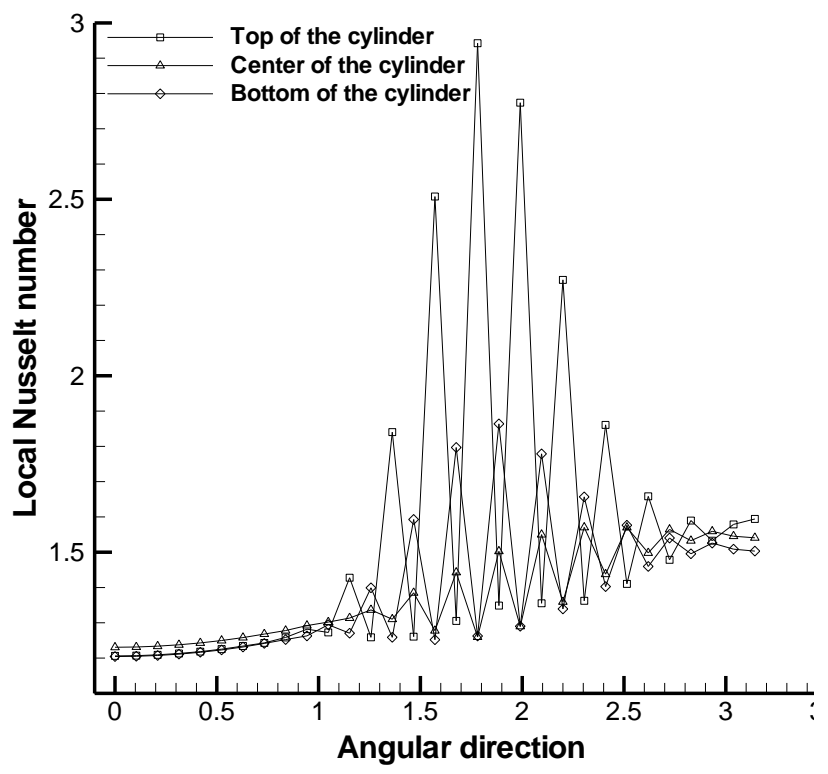

Fig.31: Variation of $\mathrm{Nu}_{\text {Local }}$ in angular direction on the cold cylinder for $\mathrm{Ra}=10, \mathrm{k}=0.87 \mathrm{~W} / \mathrm{m} \mathrm{K} \delta=0^{\circ}$

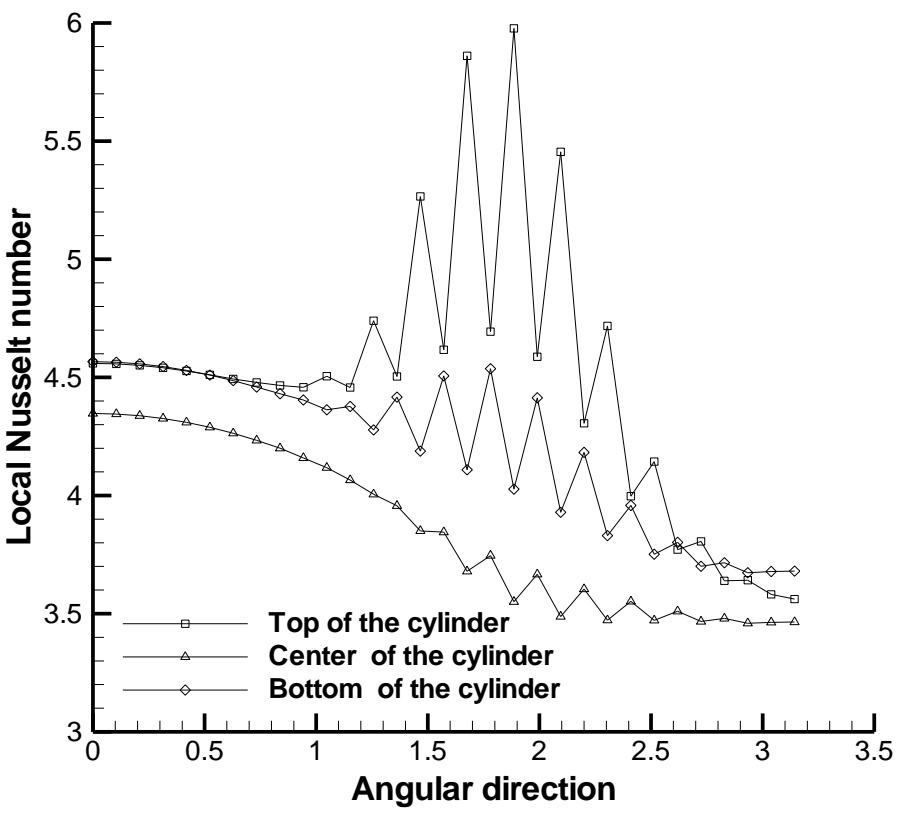

Fig.32: Variation of $\mathrm{Nu}_{\text {Local }}$ in angular direction on the hot

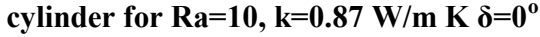

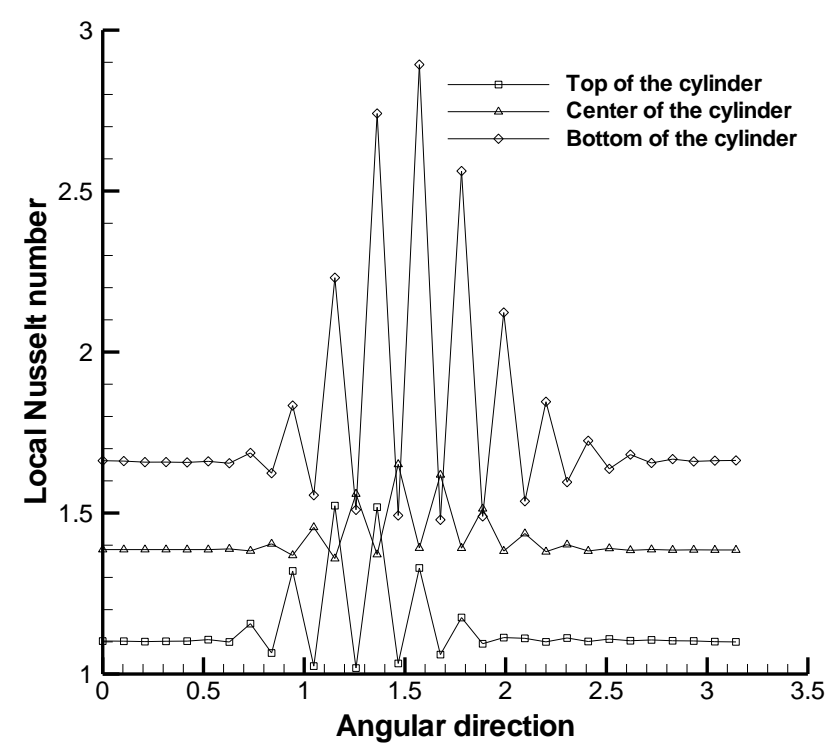

Fig.33: Variation of $\mathrm{Nu}_{\text {Local }}$ in angular direction on the cold cylinder for $\operatorname{Ra}=10, k=0.87 \mathrm{~W} / \mathrm{m} \mathrm{K} \delta=90^{\circ}$ 


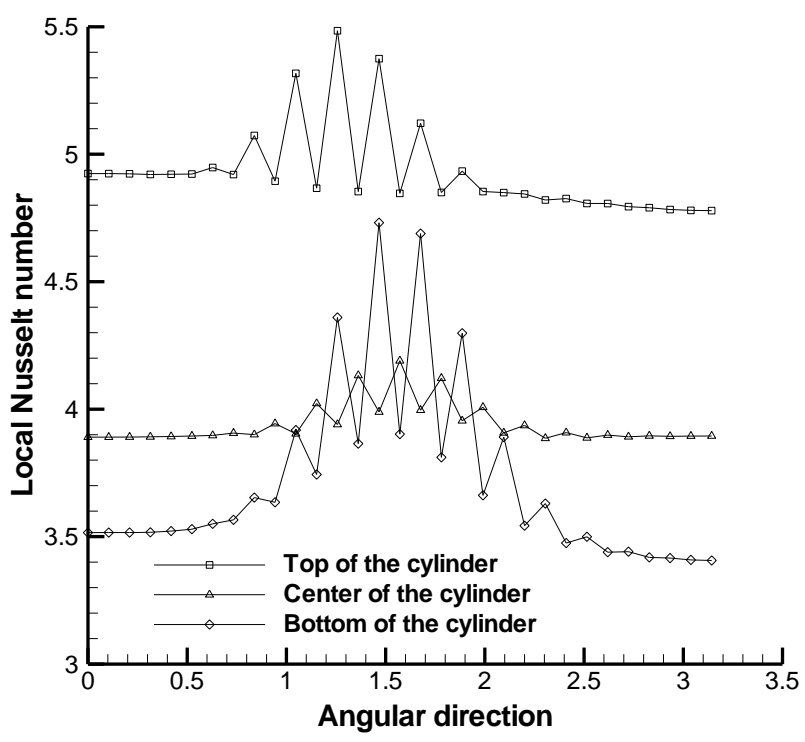

Fig.34: Variation of $\mathrm{Nu}_{\text {Local }}$ in angular direction on the hot cylinder for $\mathrm{Ra}=10, \mathrm{k}=0.87 \mathrm{~W} / \mathrm{m} \mathrm{K} \delta=90^{\circ}$

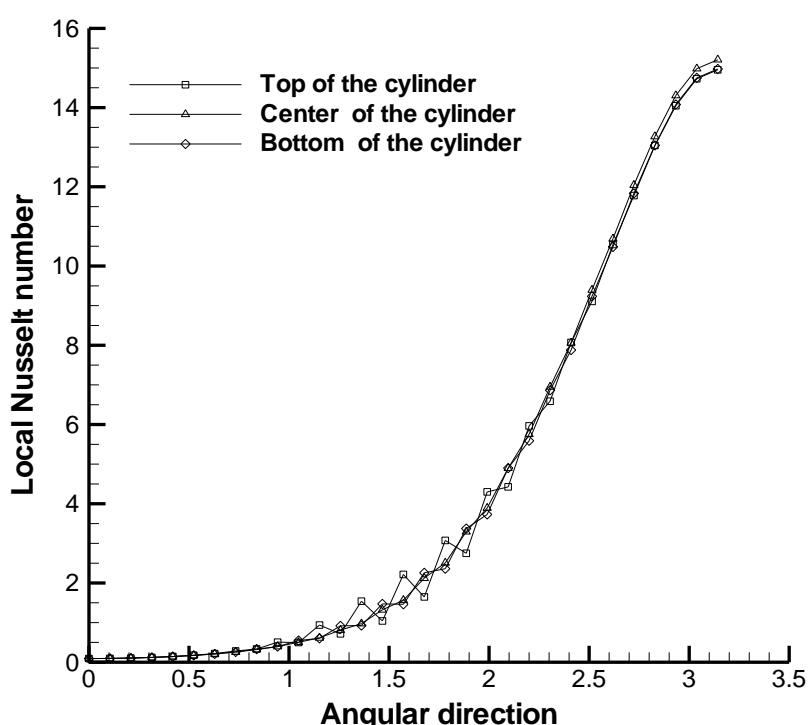

Fig.35: Variation of $\mathrm{Nu}_{\text {Local }}$ in angular direction on the cold cylinder for $\mathrm{Ra}=500, \mathrm{k}=\mathbf{0 . 8 7} \mathrm{W} / \mathrm{m} \mathrm{K} \delta=0^{\circ}$

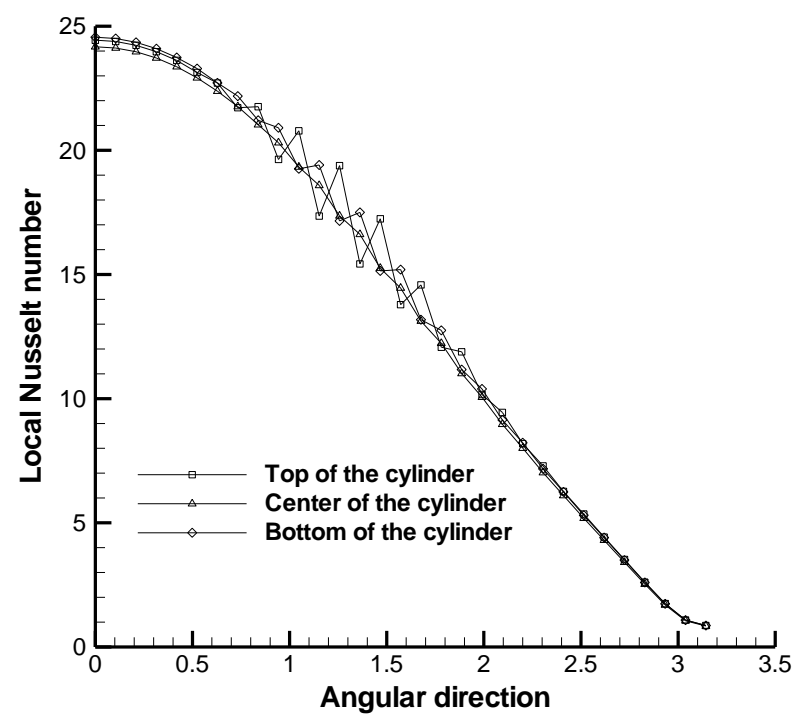

Fig.36: Variation of $\mathrm{Nu}_{\text {Local }}$ in angular direction on the hot cylinder for $\mathrm{Ra}=500, \mathrm{k}=\mathbf{0 . 8 7} \mathrm{W} / \mathrm{m} \mathrm{K} \delta=0^{\circ}$

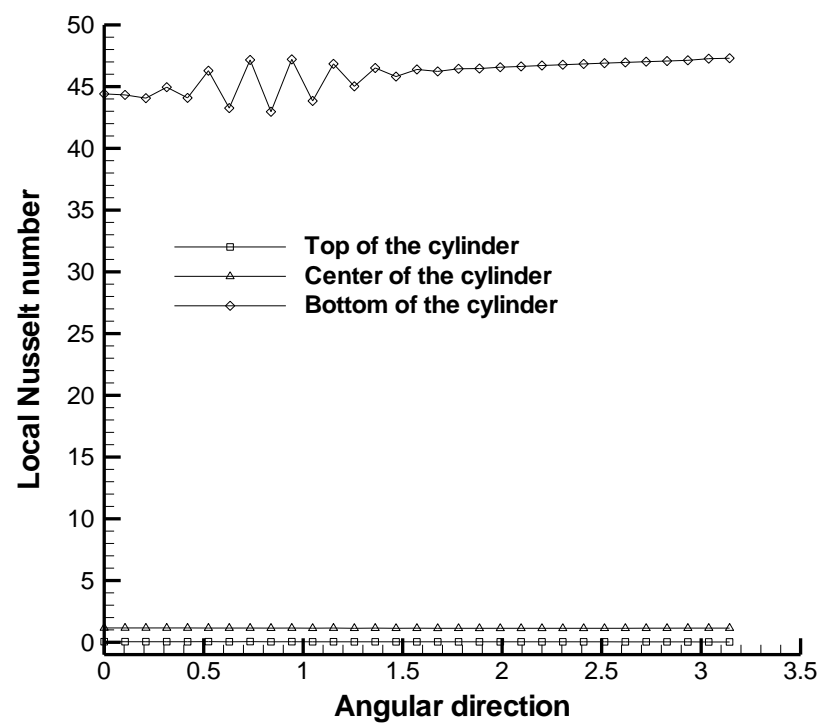

Fig.37: Variation of $\mathrm{Nu}_{\mathrm{Local}}$ in angular direction on the cold cylinder for $\mathrm{Ra}=500, \mathrm{k}=0.87 \mathrm{~W} / \mathrm{m} \mathrm{K} \delta=90^{\circ}$ 


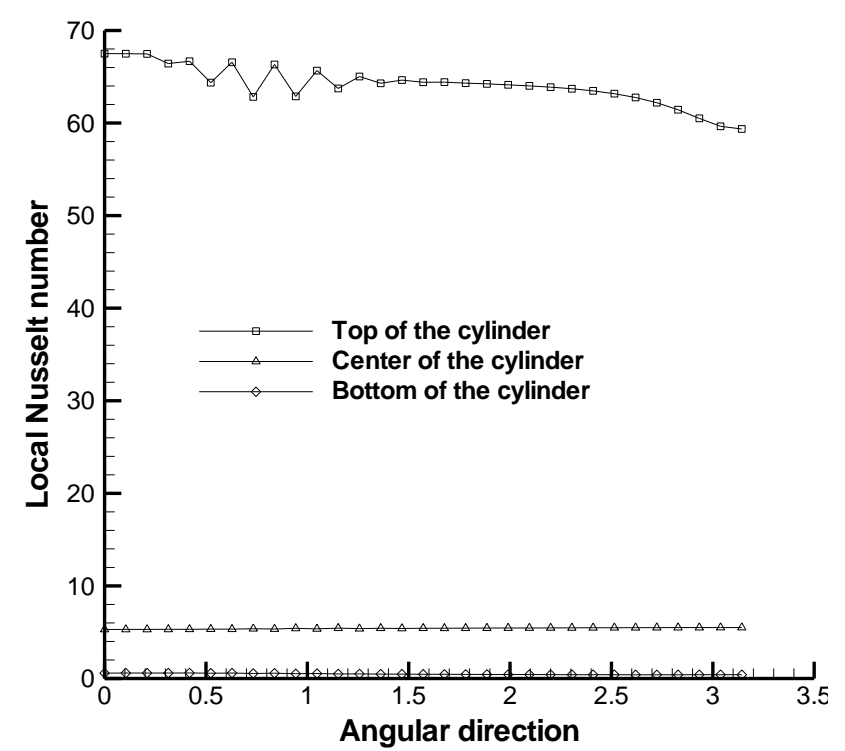

Fig.38: Variation of $\mathrm{Nu}_{\text {Local }}$ in angular direction on the hot

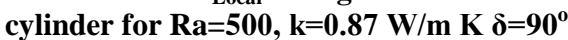

Correlations were deduced from the numerical data which are given as:

For k=0.87 W/m K:

$$
N u_{\text {out }}=\frac{0.746 R a^{0.23}}{\delta^{9.955}}
$$

For $\mathrm{k}=11.1 \mathrm{~W} / \mathrm{m} \mathrm{K}$ :

$$
N u_{\text {out }}=\frac{0.862 R a^{0.216}}{\delta^{8.1}}
$$

\section{CONCLUSIONS}

The following major conclusions can be drawn from the study:

1- The deviation between the average $\mathrm{Nu}$ for the maximization and minimization of the thermal conductivity is equal to $5.1 \%$ for horizontal annulus $\delta=0^{\circ}$ and $10 \%$ for vertical annulus $\delta=90^{\circ}$. For the inner hot cylinder the deviation between the two cases is not significant for $\delta=0^{\circ}$ but it will be significant only for vertical annulus at $\mathrm{Ra}^{*}=500$ and equal to $12.8 \%$.

2- For all parameters, results showed that the average $\mathrm{Nu}$ number decrease with the increase of $\delta$ for high values of $\mathrm{Ra}^{*}$ and increases with an increase in modified Rayleigh number but for low values of $\mathrm{Ra}^{*}$, the effect of $\delta$ on the average $\mathrm{Nu}$ will be low.

3- Local $\mathrm{Nu}$ increases in the angular direction for the outer cold cylinder and has a trend to decrease on the inner hot cylinder.

4- The maximum value of the local Nusselt number at vertical position $\left(\delta=90^{\circ}\right)$ of the annulus.
5- for $\mathrm{k}=0.87 \mathrm{~W} / \mathrm{m} \mathrm{K}$ (perpendicular direction of fibers), the region in the bottom is colder than that for the case of $\mathrm{k}=11.1 \mathrm{~W} / \mathrm{m} \mathrm{K}$ (parallel direction of fibers) for the same values of $\mathrm{Ra}^{*}$ and $\delta$.

6- The streamlines have high intensity in the boundaries and at the bottom and as $\delta$ increase to $30^{\circ}, 45^{\circ}, 60^{\circ}$ and $90^{\circ}$ the center of the streamlines of negative values expand and fade away to the lower region

\section{NOMENCLATURE

\begin{tabular}{|c|c|c|}
\hline$C_{p}$ & $\begin{array}{l}\text { Specific heat at constant } \\
\text { pressure }\end{array}$ & $\mathrm{kJ} / \mathrm{kg}^{\circ}$ \\
\hline $\mathrm{g}$ & Acceleration due to & $\mathrm{m} / \mathrm{s}^{2}$ \\
\hline $\mathrm{H}_{\mathrm{f}}$ & Fin length & $\mathrm{m}$ \\
\hline$k_{f}$ & $\begin{array}{l}\text { Thermal conductivity of the } \\
\text { fluid }\end{array}$ & $\mathrm{W} / \mathrm{m} \mathrm{K}$ \\
\hline$k_{s}$ & $\begin{array}{c}\text { Thermal conductivity of the } \\
\text { solid }\end{array}$ & $\mathrm{W} / \mathrm{m}$ \\
\hline$K_{\text {eff }}$ & $\begin{array}{l}\text { Effective thermal conductivity } \\
\text { of the porous media }\end{array}$ & $\mathrm{W} / \mathrm{m}$ \\
\hline$K$ & Permeability & $\mathrm{m}^{2}$ \\
\hline$l$ & Cylinder length & $\mathrm{m}$ \\
\hline$L$ & Dimensionless cylinder length & - \\
\hline$N u_{l}$ & $\begin{array}{l}\text { Local Nusselt number on the } \\
\text { inner cylinder }\end{array}$ & - \\
\hline$N u_{2}$ & $\begin{array}{l}\text { Local Nusselt number on the } \\
\text { outer cylinder }\end{array}$ & - \\
\hline$N u_{\text {in }}$ & $\begin{array}{l}\text { Average Nusselt number on the } \\
\text { inner cylinder }\end{array}$ & - \\
\hline$N u_{\text {out }}$ & $\begin{array}{l}\text { Average Nusselt number on the } \\
\text { outer cylinder }\end{array}$ & - \\
\hline $\mathrm{p}$ & Pressure & $\mathrm{N} / \mathrm{m}^{2}$ \\
\hline$R$ & Dimensionless radial coordinate & $\mathrm{m}$ \\
\hline$R a^{*}$ & Modified Rayleigh number & - \\
\hline$S$ & Fin pitch & $\mathrm{m}$ \\
\hline$T$ & Temperature & K \\
\hline & velocity component in $\mathrm{r}, \phi$ and $\mathrm{z}$ & $\mathrm{m} / \mathrm{s}$ \\
\hline
\end{tabular} 8.1. Latin Symbols}
$U_{n}, U_{\phi}, U_{z} \quad$ Dimensionless velocity component in $\mathrm{R}, \phi$ and $\mathrm{Z}$ direction
Z Dimensionless axial coordinate

\subsection{Greek Symbols}

$\begin{array}{ccc}\theta & \text { Dimensionless temperature } & - \\ \varphi & \text { Volume fraction } & - \\ \mu_{f} & \text { Dynamic viscosity of fluid } & \text { Pa.s } \\ \alpha_{e f f} & \text { Effective thermal diffusivity } & \mathrm{m}^{2} / \mathrm{s} \\ \alpha_{m} & \text { Medium thermal diffusivity } & \mathrm{m}^{2} / \mathrm{s} \\ \beta & \text { Volumetric thermal expansion } & 1 / \mathrm{K} \\ & \text { coefficient } & \\ \delta & \text { Angle of inclination } & \text { degree } \\ \psi_{r}, \psi_{\phi}, \psi_{z} & \text { Vector potential component in } & -\end{array}$




\section{REFERENCES}

[1] Yuan Bo, Shuqiang Ding, Dongdong Wang, Gang Wang, Hongxia Li, 2012, Heat insulation properties of silica aerogel/glass fiber composites fabricated by press forming, Materials Letters 75, 204-206.

[2] Hubert Jopek and Tomasz Strek, 2011, Optimization of the effective thermal conductivity of a composite, Convection and Conduction Heat Transfer, Poznan University of Technology, Institute Of Applied Mechanics Poland, 17, October, 197-214.

[3] Carola Esposito Corcione*, Alfonso Maffezzoli, 2013, Transport properties of graphite/epoxy composites: Thermal, permeability and dielectric characterization, Polymer Testing 32, 880-888.

[4] Gaosheng Wei, Yusong Liu, Xinxin Zhang, Fan Yu, Xiaoze Du, 2011, Thermal conductivities study on silica aerogel and its composite insulation Materials, International Journal of Heat and Mass Transfer 54, $2355-2366$.

[5] Wang H.M., Liu C.B., 2013, Analytical solution of twodimensional transient heat conduction in fiber-reinforced cylindrical composites, International Journal of Thermal Sciences 69, 43-52.

[6] Sangwook Sihn, Sabyasachi Ganguli, David P. Anderson, Ajit K. Roy, 2012, Enhancement of through-thickness thermal conductivity of sandwich construction using carbon foam, Composites Science and Technology 72, 767-773.

[7] Jun-Jie Zhao, Yuan-Yuan Duan, Xiao-Dong Wang, BuXuan Wang, 2012, Radiative properties and heat transfer characteristics of fiber-loaded silica aerogel composites for thermal insulation, International Journal of Heat and Mass Transfer 55, 5196-5204
[8] Gaosheng Wei, Yusong Liu, Xinxin Zhang, Xiaoze Du, 2013, Radiative heat transfer study on silica aerogel and its composite insulation materials, Journal of NonCrystalline Solids 362 (2013) 231-236.

[9] Nield D. A. and Bejan A., "Convection in Porous Media", Springer-Verlag, New York, 1999.

[10] Fukuda K., Takata Y., Hasegawa S., Shimomura H. and Sanokawa K., 1980, Three - Dimensional Natural Convection in a Porous Medium Between Concentric Inclined Cylinders, Proc. $19^{\text {th }}$ Natl Heat Transfer Conf., Vol. HTD -8, $97-103$.

[11] Wang Bu - Xuan and Zhang Xing, 1990, Natural Convection in Liquid Saturated Porous Media Between Concentric Inclined Cylinders, Int. J. Heat and Mass Transfer Vol. 33. No 5, 827-833.

[12] Ramón L. F. and Sergio G. M., 2007, Three Dimensional Natural Convection in Finned Cubical Enclosure, Int. J. of Heat and Fluid Flow. Vol. 28,289-298.

[13] Aziz K. and Hellums J. D., 1967, Numerical Solution of the Three Dimensional Equations of Motion for Laminar Natural Convection, The Physics of Fluids, V01. 10, No. $2,314-324$.

[14] Saad M. AL-Mashaat, Manal H. AL-Hafidh, 2012, Parametric Study of Laminar Free Convection in Inclined Porous Annulus with Fins on the Inner Cylinder, Journal of Engineering, Number 5 Volume 18 May 2012

[15] Kayhani M. H., Shariati M., Nourozi M., Karimi Demneh M., 2009, Exact Solution of Conductive Heat Transfer in Cylindrical Composite Laminate, Heat Mass Transfer 46, 83-94. 